\title{
Poorly performing point-of-care tests for chlamydia: what can be done?
}

\section{Sue Skidmore \\ EDITOR'S \\ CHOICE}

van Dommelen et al (see page 355) convincingly show the problems associated with some Chlamydia trachomatis point-of-care (POC) tests that have concerned many of us for some time. ${ }^{2}$ They have looked at three commercially available Conformitée Européene (CE)marked kits and compared the results with the current 'gold standard', a nucleic acid amplification test (NAAT). Testing was carried out in what might be regarded as optimal conditions by healthcare professionals and even so the authors describe 'alarmingly poor performance', particularly in terms of sensitivity and positive predictive value. The results obtained would have led to infections being missed and to those with falsepositive results being treated unnecessarily. With growing awareness of the development of antimicrobial resistance this latter is worrying. The results also highlight the fact that a CE mark is not a guarantee of diagnostic accuracy. CE marking is a declaration by a manufacturer that a product meets the requirements of the relevant legislation implementing certain European directives. $^{3}$ To obtain a CE mark manufacturers are required to demonstrate that their test is comparable to those already CE marked. Depending on the test used as comparator, this does not ensure the most up to date standards for sensitivity and specificity.

Correspondence to Dr Sue Skidmore, Department of Microbiology, Princess Royal Hospital, Telford TF1 6TF, UK; sue.skidmore@sath.nhs.uk
Furthermore, the presentation style of these performance data in the manufacturer's product description varies widely. The value of a CE mark would be enhanced by the introduction of a prescribed format for presentation of sensitivity and specificity. This should include both results for comparison of individual sample types and results in terms of diagnosing 'infected patient' status.

There is no doubt that a highly sensitive and specific POC test for sexually transmitted infections would be a useful tool in enabling diagnosis and treatment in one visit, thereby reducing onward transmission. In recognition of this, as stated, WHO has developed a set of criteria by which POC tests should be judged (ASSURED). The authors also point out that guidelines for the evaluation of POC tests do exist and should be adhered to. Tests to rival the NAAT 'gold standard' are now in development and will, hopefully, soon be available and be eligible for this stringent evaluation process.

However, of perhaps even greater concern is the issue of self-testing. It should be made clear that there is a difference between self-sampling kits in which a sample is usually sent to a laboratory for testing and self-testing kits in which the clients carry out the test themselves. In the case of the former, if the test employed is a NAAT then there can usually be some degree of confidence in the result. At least one of the poorly performing tests described here is available on the internet for home use. With home tests, not only is there concern about the performance of the tests, but there also have to be reservations about the competency of the general public to carry out and interpret the results of self-tests, particularly with respect to treatment and partner notification. ${ }^{4}$ In addition, even when information is supplied, do clients act on the result obtained? This question remains to be answered. A recent study looked at services available over the internet and reported disappointing results in terms of information and home tests with poor accuracy, thus reinforcing the conclusions reported here. ${ }^{5}$

So, in conclusion, it is widely agreed that rapid tests for sexually transmitted infections with high sensitivity and specificity would provide advantages, although the introduction of their use, particularly for home use, needs to be rigorously evaluated and controlled with an assurance that robust quality assurance is in place when appropriate.

\section{Competing interests None declared.}

Contributors SS is the sole contributor to this article.

Provenance and peer review Commissioned; not externally peer reviewed.

Accepted 18 July 2010

Sex Transm Infect 2010;86:330.

doi:10.1136/sti.2010.044719

\section{REFERENCES}

1. van Dommelen $\mathbf{L}$, van Tiel FH, Ouburg $\mathrm{S}$, et al. Alarmingly poor performance in Chlamydia trachomatis point-of-care testing. Sex Transm Infect 2010;86:355-9.

2. Gaydos CA. Can we climb out of the 'pit' of poorly performing rapid diagnostic tests for chlamydia? Sex Transm Infect 2009;85:158.

3. The CE Mark Bulletin no. 2. http://www.mhra.gov.uk/ home/groups/es-era/documents/publication/ con007490.pdf (accessed Sep 2007).

4. Skidmore S, Randall S, Mallinson H. Testing for Chlamydia trachomatis: self-test or laboratory-based diagnosis? J Family Plann Reprod Health Care 2007:33:231-2.

5. Owens SL, Arora N, Quinn N, et al. Utilising the Internet to test for sexually transmitted infections: results of a survey and accuracy testing. Sex Transm Infect 2010;86:112-16. 